\title{
In situ Ba isotopes analyses in barites using LA-MC-ICP-MS
}

\author{
SHENGHUA ZHANG ${ }^{*}$, MiNG Li, WEN ZHANG ${ }^{1}$, TAO LUO, \\ QIAN NI, ZHAOCHU HU \\ State Key Laboratory of Geological Processes and Mineral \\ Resources, China University of Geosciences, Wuhan \\ 430074, China; \\ *Correspondence to: zhanghaoyhsina@outlook.com
}

Barite is the main occurrence mineral of barium in nature, mainly produced in hydrothermal deposits. The composition of $\mathrm{Ba}$ isotope in barite can indicate the origin of barite deposits[1]. In this study, we present the first high precision method to analyze stable $\mathrm{Ba}$ isotope ratios in barite using laser ablation multiple collector inductively coupled plasma mass spectrometry (LA-MC-ICP-MS). The powder of barium sulfate standards (NBS127, IAEA-SO-5, IAEA-SO-6) were sintered at high temperature and pressure with the multi-anvil apparatus to form standard substance pellets for in-situ determination of $\mathrm{Ba}$ isotope. The analysis mode of small laser spot sizes $(16-44 \mu \mathrm{m})$ and low laser ablation frequency $(1 \mathrm{~Hz})$ combined with a "wave" signal-smoothing device was used. The obtained analytical precision was significantly improved by using the signal-smoothing device in comparison with that without the signal-smoothing device. NBS127 pellet was selected as the external reference material for matrix-matched calibration. Two barium sulfate standard materials (IAEASO-5, IAEA-SO-6) and four natural barite samples (Ba-Fra, Ba-FJ, Ba-YN, Ba-HN) were analyzed. Typical intermediate precisions for $\delta^{137 / 134} \mathrm{Ba}$ was $0.15 \%$ (2SD, standard deviation). The determined $\delta^{137 / 134} \mathrm{Ba}_{\mathrm{NBS} 127}$ values of barium sulfate standard materials (IAEA-SO-5, IAEA-SO-6) and four natural barite samples by LA-MC-ICP-MS were consistent with recommended values analyzed by double spike method combined with solution MC-ICP-MS. Barium sulfate standard material NBS127 as well as two natural barite samples (Ba-Fra, Ba-FJ) were recommand as the potential standards for in situ $\mathrm{Ba}$ isotope ratios analysis by LA-MCICP-MS.

[1] Crockford et al. (2019) EPSL. 519, 234-244. 